\title{
Measurement of impurities to support process development and manufacture of biopharmaceuticals
}

Review for Trends in Analytical Chemistry special issue: Control of Impurities

Authors: Sheun Oshinbolu1,2, Louisa J Wilson¹,2, Will Lewis², Rachana Shah², Daniel G Bracewell $^{1}$

${ }^{1}$ Dept of Biochemical Engineering, University College London, Bernard Katz Building, Gordon Street, London, WC1H 0AH, United Kingdom

${ }^{2}$ GlaxoSmithKline, Gunnels Wood Road, Stevenage, Herts, SG1 2NY, United Kingdom

Corresponding Author:

Prof. Daniel G. Bracewell, Dept of Biochemical Engineering, University College London, Bernard Katz Building, Gordon Street, London, WC1H 0AH, United Kingdom

Email: d.bracewell@ucl.ac.uk 


\begin{abstract}
The biopharmaceutical industry is a growing market relying on analytical methods to bring safe and efficacious drugs from conception to market. Analytical methods are required during the entire drug life cycle as it plays a vital role during decision making. Impurities influence product quality, stability and efficacy, so their levels need to be minimised. There are regulatory guidelines that set out principles for consideration in meeting product quality specifications. Analytical methods have limits in their sensitivity and accuracy of measurement, their sample throughput and/or range of detection. This highlights the need to have orthogonal assays that can confidently quantify critical impurities to the appropriate levels. In this review, we discuss three major analytically challenging critical quality attributes - two product related impurities (aggregates and incorrect glycosylation) and one process related impurity (host cell proteins) - and the analytical assays that are commonly used to measure their quantity and quality.
\end{abstract}

\title{
Keywords
}

Recombinant therapeutics, aggregation, host cell proteins, glycosylation, regulation, biopharmaceuticals

\section{Abbreviations}

2D-PAGE - Two-dimensional polyacrylamide gel electrophoresis

ADCC - antibody-depedent cell-mediated cytotoxicity

AUC / SV-AUC - (Sedimentation velocity) analytical ultra-centrifugation

$\mathrm{BiP}$ - Binding immunoglobulin protein

CD - Circular dichroism

$\mathrm{CHO}$ - Chinese hamster ovary

CPP - Critical process parameter

CQA - Critical quality attribute

Cryo-EM - Electron cryo-microscopy

DLS - Dynamic light scattering

ELISA - Enzyme linked immunosorbent assay

ER - Endoplasmic reticulum

FDA - Food and Drug Administration

HCCF - Harvested cell culture fluid

HCP - Host cell protein

ICH - International Council for Harmonisation

IgG - Immunoglobulin G

LC - Light chain (of an antibody)

$\mathrm{mAb}$ - Monoclonal antibody 
MS / MS-MS / LC-MS / HDX-MS - Mass spectrometry / Tandem mass spec / Liquid chromatography with mass spec / Hydrogen-deuterium exchange mass spec

MFI - Micro-flow imaging

NMR - Nuclear magnetic resonance

NTA - Nanoparticle tracking analysis

PLBL2 - Phospholipase B-Like 2

ppm - parts per million

PTM - Post-translational modifications

QbD - Quality by design

QC - Quality control

SAXS - Small-angle X-ray scattering

SEC - Size exclusion chromatography

TEM - Transmission electron microscopy

UPLC - Ultra performance liquid chromatography

UV - Ultra violet (light) 


\section{Introduction}

Recombinant therapeutic antibodies are a major player in today's pharmaceutical industry, with biopharmaceuticals representing $24 \%$ of the global drug market and antibody derived drugs reaching sales of $\$ 82$ billion in 2016 [1]. Half of the top 10 best-selling drugs are monoclonal antibodies (mAbs) [1] and they are mostly used to treat cancer and autoimmune diseases. Some recombinant therapeutics require post-translational modifications (PTMs) such as glycosylation for clinical efficacy. Hence, mammalian host expression systems that can make these modifications (such as Chinese hamster ovary (CHO) cells) are typically used [2]. However, microbial expression systems (e.g. E.coli) are still the preferred choice for biological medicines such as insulin that don't require PTMs [2].

In order to have a full understanding of the molecule being produced, analytical methods are required from discovery and process development through to clinical trials. As such analytics play a vital role in taking recombinant therapeutics from bench-to-market as it underlies all decision making. To achieve consistency in production, quality by design (QbD) is becoming a globally accepted strategy within the industry with the goal of enhancing pharmaceutical manufacture through design and control of processes [3]. The strategy systematically establishes critical quality attributes (CQA) of a drug product and critical process parameters (CPP) which are parameters with significant impact to CQAs [4]. CPPs and CQAs are used to create a design space in order to develop a control strategy. Three CQAs that have proven to be particularly challenging to monitor and control are aggregated product, incorrectly glycosylated product and host cell proteins. In this review, we will therefore discuss the importance of analytical assays and how regulatory guidelines aid to control these three CQAs.

\section{Regulatory guidelines}

The International Council for Harmonisation of Technical Requirements for Pharmaceuticals for Human Use (ICH) publishes scientific guidelines that are harmonised between the regulatory authorities and the pharmaceutical industries in Europe, Japan and the United States of America. The ICH Q6B guideline sets out the principles for consideration in setting product quality specifications (appearance, identity, purity and impurities, potency and quantity) as well as appropriate analytical procedures. Similar guidelines on the quality, safety, and efficacy of biotherapeutic protein products prepared by recombinant DNA technology are provided by the World Health Organization [5]. These guidelines help to ensure the commercialisation of 
only consistent, effective, high quality products that undergo a highly-regulated development process. To ensure these standards are being met, an extensive analytical toolbox is required, to enable the use of a wide range of analytical techniques that target different physicochemical properties of the product [5]. The assays and instruments are expected to be precise, reproducible, robust, and commonly orthogonal techniques are carried out to complement and provide confidence in results. Fast analytical methods that can produce large amount of data have become vital to speed up time-lines and the decision-making process.

For efficient quality control, critical quality attributes (CQAs) are identified and closely monitored. A CQA as outlined in ICH Q8 (R2) is a "physical, chemical, biological or microbiological property or characteristic that should be within an appropriate limit, range, or distribution to ensure the desired product quality" [6]. Figure 1 outlines the key steps towards controlling CQAs, which can be product or process related. Product related impurities are molecular variants of the product such as aggregates, fragments, incorrectly glycosylated antibody or charge variants, whereas process related impurities are an inherent part of the process such as the host cells' DNA or host cell proteins (HCPs), leachables (such as protein A) and viruses. The presence of these impurities in the final drug product can affect product purity, product efficacy and stability, and can cause adverse immune responses in patients such as anaphylaxis [7]. To ensure patient safety, impurities need to be reduced to acceptably low levels to meet the defined acceptance criteria. For mAbs, the final product should typically have $<5 \%$ high molecular weight aggregates, $<100$ ppm HCPs and $<10 \mathrm{ng} /$ dose DNA $[8,9]$, although in reality aggregation and HCP limits are case-by-case dependent and are defined from (pre-)clinical studies and manufacturing consistency lots [10].

\section{Analytics during the drug lifecycle}

Analytical methods are essential from drug discovery through to clinical trials, and even after regulatory approval. They are needed to ensure full characterisation of the molecule, however the extent of analytics used differs throughout the product lifecycle. At the earlier stages of the lifecycle (drug discovery and development) detailed characterisation is required to understand the product and how different process conditions change the product profile, so that we can define the manufacturing strategy. Comparability studies as addressed in the ICH Q5E guideline might also be required. By contrast, once the manufacturing process is defined and 
controlled, it might not be necessary to perform extensive quality control (QC) at later stages. In particular, for manufacturing lots that are released for clinical studies, it may be acceptable to select only a subset of appropriate assays, since the production process should be tightly controlled and consistent at this stage [10].

It should be noted that single assays do not always provide a true representation of a molecule. Biopharmaceuticals are complex molecules which are constantly moving in solution. Some analytical techniques allow protein samples to be measured in the native state while others measure proteins in a non-native state conformation. In this sense, some analytical methods can be destructive, meaning either that sample treatment irreversibly denatures the protein, preventing the sample to be reused for other means, or that the sample is consumed during the measurement. Non-destructive methods are typically preferred as this allows a single sample to be reused for other analytical methods.

Figure 2 shows a typical bioprocess flow diagram for the production and purification of biopharmaceuticals (upstream and downstream processing respectively) and highlights the key stages at which assays are required to demonstrate efficient impurity clearance. Advances in the measurement of three typical CQAs of importance that will be discussed in this review are aggregation, glycosylation and HCPs. Aggregates can form during the protein synthesis as well as during product purification while on the chromatography column, and therefore need to be tightly controlled. Incorrect glycosylation of proteins can severely affect efficacy and stability of the drug substance, and HCPs can likewise affect the product stability, but more importantly could pose severe immunological risks to the patients if not reduced to acceptably low levels. Since these three CQAs have proven to be particularly challenging to monitor and remove from the final drug product, we will focus on them in this review, although it should be noted that there are many more potential CQAs.

\section{Aggregation}

Aggregation is the self-association of protein molecules and can differ in morphology, solubility, structure, reversibility and intermolecular bonding. Moussa et al. [11] reviewed the issues of aggregated therapeutic proteins causing adverse immune responses in patients. The adverse immune response can cause problems for both patient safety and product efficacy [7]. 
Therefore, in order to ensure patient safety, it is important to improve our understanding of protein aggregates and use the most appropriate analytical techniques to monitor aggregate content. Molecular weight, structure and solubility are the three main features used to characterise aggregates. As there are different sizes and different mechanisms of aggregation, aggregates can be difficult to characterise with one single technique. Figure 3A summaries the key advantages of the different techniques used for measuring aggregates.

\subsection{Analytics for aggregation quantification and sizing}

Size exclusion chromatography (SEC) is the most common technique to quantify and size soluble aggregates less than $50 \mathrm{~nm}$ (Figure 3B). It is the most typically used technique of ensuring that the amount of mAb aggregates meet regulatory guidelines $(<5 \%)$. It is a robust and well-established technique with separation on the column occurring based on size. Larger molecules which have less accessibility to the pores elute first. SEC combined with multi-angle light scattering (MALLS) can provide conformation of the average molecular weight of species. SEC-MALLS is also more sensitive to higher order aggregates which may not be detected by SEC alone [12]. SEC is not suited to analyse insoluble or large aggregates as they often get trapped by frits which protect the column. Additionally, the mobile phase dilutes samples from initial loading concentration, which can cause weakly associated aggregates to dissociate. Therefore, it is important to bear in mind when analysing results that SEC may not be a true representation of a sample. Asymmetrical Flow Field Fraction (AF4) is an alternative analytical technique which can quantify larger aggregates than SEC (even insoluble aggregates). However, it is not as mature as SEC and requires in-depth method development[13].

Dynamic light scattering (DLS) is commonly used in industry to estimate the size and relative percentage of aggregate species. The scattering of light depends on the molecular weight, concentration and shape of the molecule, as well as the light/laser wavelength and scattering angle [14]. DLS is more capable of measuring larger aggregates across a wider sample concentration range $(0.1-50 \mathrm{mg} / \mathrm{ml})$ than SEC [15]. As intensity of light is proportional to molecular weight, the technique is highly sensitive but often biased to large aggregates as they scatter light more strongly. The technique is poor at resolving small oligomers such as dimers and trimers as it requires a three to four fold [15] difference in hydrodynamic size to resolve different species. Techniques such as nanoparticle tracking analysis (NTA) and micro-flow 
imaging (MFI) can characterise larger aggregates and particulates which are important to be aware of, especially when the molecule is in the final formulation. NTA is employed for sizing submicron particles $(10 \mathrm{~nm}-1 \mu \mathrm{m})$ whereas MFI is for micro particles $(1-100 \mu \mathrm{m})[16]$. Both techniques enable sample visualisation, however NTA is less reproducbile and requires several optimisation steps by a skilled operator. NTA is also very sensitive to the buffer which can raise the interference from the background and make it difficult for the software to track aggregates. MFI requires large amounts of sample and high shear forces can cause particle fragmentation [17].

Sedimentation velocity analytical ultra-centrifugation (SV-AUC) uses high centrifugal speeds to study sedimentation behaviour. Radial separation causes larger species to sediment to greater extent than smaller molecules. AUC covers the intermediate size between SEC and DLS with excellent separation and detection for low level aggregates $\leq 1 \%$ [18]. Berkowitz [19] showed comparable dimer levels to SEC in samples with a range of dimer content. The major limitation with AUC is its low throughput, as it takes 12 hours to run a single sample. Samples are measured in the native state and although it is not completely destructive it is not recommended to use for other aggregation assays. Analysis of data is also time consuming and requires operators with expert experience. Although AUC is more expensive than SEC and DLS and requires regular calibration and intensive maintenance, it is commonly used to validate a SEC method.

Overall, not a single technique can provide information on quantification and size estimation. Although SEC is a gold standard in the industry, both AUC and DLS serve as orthogonal techniques to SEC as they can detect aggregates not characterised by SEC and thus increase confidence in data interpretation.

\subsection{Analytics for structural analysis of aggregates}

Structural analysis can give insight into the mechanism of formation as well as the state of the protein (native or non-native). There are several techniques that can structurally characterise proteins [20] but for this review the following methods will be discussed: circular dichroism (CD), hydrogen-deuterium exchange mass spectrometry (HDX-MS), cross-linked mass spectrometry, small-angle x-ray scattering (SAXS), transmission electron microscopy (TEM) and electron cryo-microscopy (Cryo-EM) and fluorescence spectroscopy. 
$\mathrm{CD}$ has become recognised as a valuable structural technique to provide information on secondary and tertiary structure. CD measures the difference between the absorption of left and right circularly polarised light [21]. Operating in the far-UV (180-260 nm) provides quantitative estimates on the secondary structure (e.g. percentage helix, turns and sheets), whereas operating in the near-UV (240-340 nm) regions provides quantitative estimates on tertiary structure conformation [14]. CD is easy to perform and offers speed and convenience in comparison to NMR and X-ray crystallography as it requires low sample concentration, does not require crystallised samples and samples can be recovered [22]. However, the technique provides low resolution and sensitivity for mAbs. Historically, CD has been primarily used to investigate the secondary structure (alpha helix, beta sheets, etc.) for small molecules/proteins. The technique appears to be more sensitive to changes in alpha helices than beta sheets. Large molecules such as mAbs are primarily comprised of many beta sheets. Therefore, CD lacks the sensitivity and resolution to measure small and specific secondary structural changes as the CD outputs are averaged out.

For larger molecules, there are emerging complementary technologies which can provide greater resolution to understand structure related aggregation pathways. Cross-linked MS consists of covalently connecting two function groups of the protein(s) to gain insight into the three dimensional structure of proteins in solution using MS [23]. MS analysis can occur with the intact protein or after enzymatic digestion. HDX-MS can pinpoint structural changes down to the primary structure [24]. It probes the exchange kinetics of hydrogen for deuterium and has been able to elucidate the aggregation mechanism for mAb dimers [25]. SAXS and TEM can measure quaternary structure. SAXS can provide structural information on shape and size at 1-2 $\mathrm{nm}$ resolution [26] and TEM has been shown to visualise mAbs aggregated on the Fc or Fab region [27].

Fluorescence spectroscopy is a highly sensitive technique for both structural characterisation and aggregation detection. Fluorescence can be measured intrinsically from naturally fluorescent amino acids (e.g. tryptophan) or extrinsically with the addition of dyes. The increased focus on measuring protein aggregation for therapeutic protein manufacturing and in disease states, has resulted in an increase in publications using dyes for protein characterisation. Measuring intrinsic vs. extrinsic fluorescence is often a trade-off between the intensity of 
fluorescence signal and invasiveness [28]. Extrinsic fluorescence can be used to monitor protein folding/unfolding and detecting small amounts of large aggregates typically undetected by SEC [29].

\subsection{Aggregation in the process environment}

During the expression of recombinant therapeutics, aggregation can occur both intracellularly during synthesis as well as in the culture medium after secretion [30]. From an extracellular perspective, the exposure of the recombinant therapeutics to physical (e.g. agitation, aeration), chemical (e.g. pH) and biological damage (e.g. proteases) can cause conformational changes. From an intracellular perspective, recombinant cells express and fold higher amounts of proteins than they normally would. Under cellular stress and/or when high amounts of proteins are expressed, chaperones in the endoplasmic reticulum (ER) such as BiP can become overwhelmed. This can prevent proper folding of the nascent proteins in a timely and precise manner. Unfolding/misfolding results in the exposure of hydrophobic patches of the peptide structures which are prone to aggregation [30,31]. Aggregates can also form during protein purification while on the chromatography column. This shows the impact that upstream and downstream conditions can have on the stability of the product, highlighting the importance of having process understanding of aggregation.

\section{Glycosylation}

Glycosylation is an important post-translational modification that occurs during protein expression in eukaryotic cells. It is the most complex post-translational modification in terms of chemical heterogeneity. Carbohydrate complexes termed glycans are added to proteins, through attachment to specific amino acids, and play a role in many functions such as solubility, stability, binding and efficacy. Glycosylation of recombinant therapeutics is dependent on the expression system and growth conditions; hence the upstream processing conditions directly impact correct glycosylation formation. Glycosylation pathways are influenced and dependent on cell culture conditions [32] as well as on cell engineering. Pande et al. [33] have shown how the addition of a small molecule ionophore can promote specific glycosylation pathways. For mAbs that rely on Fc-mediated effector function, changes in Fc glycans can affect the safety efficacy and mechanism of action [34]. One example is Reusch and Tejada [34] found evidence that afucosylated Fc glycans increase antibody-dependent cell-mediated cytotoxicity (ADCC). 
Consequently, there has been a lot of development to measure and monitor glycosylation patterns.

The ICH Q6B guideline states that "for glycoproteins, the carbohydrate content should be determined. In addition, the structure of the carbohydrate chains, the oligosaccharide pattern and glycosylation sites of the polypeptide chain should be analysed to the extent possible" [10]. The European Medicines Agency states that "glycan structure should be characterised and particular attention should be paid to their degree of mannosylation, galactosylation, fucosylation and sialylation. The distribution of main glycan structure present (often G0, G1 and G2) should be determined" [35]. To determine this, there are three different approaches of measuring glycosylation: released glycan assay, glycopeptide and intact IgG mass spectrometry (MS) analysis. Zhang et al. [36] described the different analysis approaches for structural characterisation using mass spectrometry. For detailed structural analysis, the amount of sample preparation required is often a trade-off between sample complexity and information obtained. The longer the sample preparation time (decreasing throughput), the more structural information (e.g. sites and linkages) can be obtained.

\subsection{Releasing glycans}

Glycan analysis utilises enzymes or chemicals to release glycans to allow in-depth analysis of oligosaccharide structures, glycan profiling and quantify amounts of individual monosaccharides (such as mannose, galactose and fucose). There has been progress over the last few years to automate and increase the throughput for glycan analysis. Burnina et al. described a cost effective plate based sample preparation which denatures, reduces and deglycosylates 96 samples in 90 minutes compared to days [37]. Microfludic devices have been shown to integrate glycan cleavage, purification and chromatography separation and MS detection, reducing both time and amount of sample required [38]. Liquid handling systems have also been incorporated to create an automated sample preparation platform [39].

While released glycan analysis is considered the gold standard for quantification, MS is the gold standard for qualitative characterisation of glycans. After chemical or enzymatic release, samples need to be purified which includes desalting and enrichment to isolate the protein of interest by chromatography, electrophoresis or covalent based interactions. Tandem MS (often 
quoted as MS/MS) is an alternative approach to obtain glycan identification and sequencing using a multi-step MS that can provide purification and analysis in one system [40].

\subsection{Bottom-up and top-down approach}

A second approach utilises enzymes to release glycopeptides to determine and evaluate sites of attachment of glycopeptides and heterogeneity. This is often referred to as the bottom-up approach or peptide mapping. Although enzymatic cleavage increases sample complexity and interpretation difficulty, enzymes can be immobilised allowing re-usability, automation and reduced digestion time $[41,42]$. Similar to glycan release, samples need to be purified before analysis. The third approach of analysis, the top-down approach, measures intact IgG. This allows quantitation of the main glycoforms (G0, G1 and G2) and deduction of the overall glycan composition. However, with intact IgG, small modifications or changes in the microheterogeneity often have a too small effect to be resolved using analytical techniques.

\subsection{Analysis with mass spectrometry}

MS can be used to determine amino acid sequence, locate disulphide linkages, elucidate oligosaccharide profiles and identify other post-translational modifications [43]. It is a versatile technique for glycosylation analysis as it can characterise structural changes of intact glycopeptides and glycans. Ionisation variability, suppression and difficulties in resolving isoforms with small mass differences are the major limitations of the technique. For these reasons, it is often combined with chromatographic and electrophoretic techniques, to separate isoforms prior to MS analysis.

\section{Host Cell Proteins}

The host cells that are implemented for the expression of recombinant therapeutics produce not only the desired antibody but also indigenous proteins, known as host cell proteins (HCPs). They are present in the harvested cell culture fluid (HCCF) and are process related impurities requiring separation from the product. The composition of HCPs can vary with the process conditions under which the cells are grown [44]. HCPs also differ based on whether they are mammalian, bacterial or yeast-derived (e.g. Chinese Hamster Ovary, E.coli or Pichia pastoris respectively) [45]. HCPs are a complex mixture of a huge variety of proteins with significantly diverse physicochemical properties (e.g. pI, molecular weight and degree of hydrophobicity) $[44,45]$, which makes it necessary to use multiple techniques for efficient clearance. Shukla et al. [46] have investigated two strategies to demonstrate robust HCP clearance during 
downstream purification steps. The reason why HCP removal is so essential is not only due to the possibility that they might influence the efficacy of the drug product, but also because they can cause adverse immune reactions in patients, including cross-reactivity and autoimmunity, and as such are a major safety concern [45, 47]. One notable case was published by Genentech about phase III clinical trials for the asthma drug Lebrikizumab. Material for this study was found to contain high amounts of the HCP species PLBL2 which caused a notable immunogenic response in $\sim 90 \%$ of subjects, although no adverse safety effects were observed [48].

\subsection{Analytics for HCP quantification and identification}

Enzyme-linked immunosorbent assays (ELISAs) are considered a gold standard for the quantification of HCPs, and are most commonly used to ensure the drug specification limits of HCP impurities are met. The antibodies used in such assays are produced by immunised animals such as goats or rabbits. Therefore, only HCPs with immunogenicity in the host animals will be accurately measured. For HCPs which do not cause an immune reaction in the immunized animals, there will be no specific antibody produced and so they will not be detected in the corresponding ELISA. As an ELISA is unlikely to detect $100 \%$ of HCPs, it is only semi-quantitative and does not provide absolute results about the total mass of HCPs present in a sample [9]. Moreover, this type of ELISA does not provide any information about the identity of the HCP species that are present.

The identity of HCP species can be investigated by two-dimensional polyacrylamide gel electrophoresis (2D-PAGE) and liquid chromatography with tandem mass spectrometry (LCMS/MS). Electrophoresis with sensitive protein staining enables the visualization of the proteins, and 2D electrophoresis supplies information about the distribution of HCP species by molecular weight and isoelectric point. Coupled with LC-MS/MS, these species can be identified and quantified. This approach allows for HCP species with low immunogenicity to be recognized as well. However, low abundance proteins are often masked by highly abundant ones and are less likely to be identified [49]. This is a concern when trying to quantify and identify low abundance HCPs in the presence of highly abundant antibody product. To address this issue, recent advances in mass spectrometry have enabled improvements in the specificity and dynamic range using targeted mass spectrometry techniques, e.g. using a Q-Exactive mass spectrometer which contains a quadrupole mass filter and orbitrap mass analyser for high 
resolution and accurate mass (HR/AM) measurements [50-52]. Recent research has demonstrated that it is possible to quantify HCPs down to single-digit ppm range within 1 hour for real time bioprocess development support [53]. However, it should be noted that MS-based HCP detection is limited by the software. If it's not programmed to search for certain species, then these will not be detected.

If a certain HCP species of interest needs to be identified, ELISAs using antibodies raised against a specific problematic HCP species can be carried out. Alternatively, western blots can be performed - again, either using a polyclonal mix of anti-HCP antibodies to verify the presence of HCPs in general, or using antibodies raised against a specific HCP species to identify the particular HCP species in question [49]. However, this approach is very time consuming and it can be expensive to raise antibodies against several specific types of HCPs. Additionally, only HCP species that are immunogenic and against which antibodies can be raised in the first place, will be able to be identified using this technique [49]. Table 1 summarizes the advantages and disadvantages of each analytical assay, and often it may be necessary to use several orthogonal assays to ensure confident HCP detection and characterisation.

\subsection{Significant Research to Date}

\subsubsection{HCPs co-elute with mAbs during protein A chromatography}

When it was first discovered that significant amounts of HCPs were not being efficiently separated during the antibody purification process, but were in fact retained during protein A affinity chromatography, it was unclear whether the host cell proteins co-eluted with antibodies by non-specifically binding to the resin or by associating with the bound antibodies. Nogal et al. [45] have sufficiently demonstrated that the latter was the case. They performed two protein A chromatography runs; in the first case, $\mathrm{mAb}$ free $\mathrm{HCP}$ material was loaded onto clean Protein A resin, whereas in the second case, the $\mathrm{mAb}$ free $\mathrm{HCP}$ material was loaded onto a Protein $\mathrm{A}$ column that already had antibodies bound to it. It was found that upon elution, the HCP levels were significantly higher in the second run, demonstrating that most HCPs species co-purify during protein A affinity chromatography due to their interactions with the bound antibodies.

Nogal et al. [45] also demonstrated that only select subpopulations of host cell proteins coelute with the antibody during protein A affinity chromatography. They loaded harvested cell 
culture fluid containing antibody and HCPs onto protein A resin, spiked the flow-through with purified antibody again and recycled this material for a total of five cycles. They noted that HCP levels in the flow-through samples remained fairly consistent while eluate samples from the five cycles contained rapidly decreasing levels of HCPs. They concluded that only specific subpopulations of HCPs co-elute with the antibody, which were gradually depleted from the load material.

\subsubsection{Identification of co-eluting HCP species}

Furthermore, considerable research has been done recently to identify the specific HCP species that are being retained during protein A affinity chromatography with certain antibodies. HCPs reported to be present include those that are involved in essential cell survival processes such as in translation (e.g. elongation factor 2), in protein folding (e.g. heat-shock proteins Hsp70 and Hsp90 and clusterin), and in glucose or lipid metabolism (e.g. Glyceraldehyde 3-phosphate dehydrogenase, pyruvate kinase, lactate dehydrogenase; PLBL2) [48, 54-56]. In addition, proteases such as cathepsins and serine protease HTRA1 have been identified [54-56], particularly during late stages of the cell culture process when they are suggested to cause fragmentation $[54,57]$.

\subsubsection{Effects of upstream process parameters on HCP composition}

Work has also been done to investigate whether certain upstream process conditions affect the composition of HCPs present in HCCF. For instance, Jin et al. [44] studied how media, temperature, feeding strategy, agitation speed, process duration and cell viability impact the levels of total HCPs. They found low cell viability to have the most significant effect on HCP composition. Not only did they measure higher levels of HCPs at day 15 - when viability was only $11 \%$ - but they also discovered that low molecular weight species were more abundant at this time in the fermentation process, suggesting the release of proteases and the associated degradation of proteins at low viability. This suggests that viability needs to be closely monitored, although it should be noted that the viability of $11 \%$ observed in this research is not a realistic manufacturing process viability. Tait et al. [55] have also reported that the changes in environment, metabolism and declining viability at the end of the fermentation period result in different compositions of HCPs at the end of the process compared to earlier days. Both the work from Jin et al. [44] and Tait et al. [55] suggest that the time of harvest is a crucial parameter with regards to HCP composition and that cell culture duration and cell viability should be controlled for process consistency. 


\section{Conclusion}

The increasing number of biopharmaceuticals being brought to market require a versatile analytical toolbox to verify that only safe and efficacious drugs of high quality are commercialised. Analytical methods are required throughout the entire drug life cycle - from discovery and process development through to clinical trials and final market distribution - and therefore play a vital role in success. The approach to quality specifications throughout are governed by guidelines published by the ICH and are harmonised between the regulatory authorities and the pharmaceutical industries in Europe, Japan and the United States of America. In this review, we have discussed three major CQAs and the analytical methods that are most commonly used to measure their quantity and/or quality. Aggregates and HCPs are impurities that can severely affect product quality and therefore need to be reduced to acceptably low levels. Glycosylation is an important post-translational modification that needs to be carried out correctly by the cells. Different glycosylation patterns can influence the drug solubility, stability, binding and efficacy, hence the glycosylation profile needs to be controlled. The impacts these impurities can have, show the importance of having a defined set of analytical assays to quantify the impurities in a sensitive and consistent manner. When combined with process understanding of their origin, they allow those working in process development and manufacturing to be aware of all product quality issues that may have been caused by any process changes. Looking to the future, the ultimate goal would be for analytical methods to support the development and production of therapeutic proteins in real-time. While the development of online assays still provides difficulties, it is likely that we will see more atline assays coming to fruition in the future.

\section{Acknowledgements}

This research was supported by the UK Engineering and Physical Sciences Research Council (EPSRC) grant EP/L01520X/1, the Biotechnology and Biological Sciences Research Council (BBSRC) grant BB/N503812/1, and GlaxoSmithKline.

The authors would also like to acknowledge Mike Molloy and Mitul Patel at GlaxoSmithKline for their helpful feedback. 


\section{References}

[1] Hall. M, Hill. D, P. D, Global Pharmaceuticals, 2016 industry statistics. , Hardman\&co., 2017.

[2] G. Walsh, Biopharmaceutical benchmarks 2014, Nature Biotechnology, 32 (2014) 992-1000.

[3] C. Finkler, L. Krummen, Introduction to the application of QbD principles for the development of monoclonal antibodies, Biologicals, 44 (2016) 282290.

[4] E. Ohage, R. Iverson, L. Krummen, R. Taticek, M. Vega, QbD implementation and Post Approval Lifecycle Management (PALM), Biologicals, 44 (2016) 332-340.

[5] World Health Organisation, Guidelines on the quality, safety, and efficacy of biotherapeutic protein products prepared by recombinant DNA technology, WHO Press, Switzerland, 2013.

[6] ICH, ICH Harmonised Tripartite Guidelines Pharmaceutical Development Q8 (R2) Step 4 version, 2009.

[7] FDA, Guidance for industry: immunogenicity assessment for therapeutic protein products, 2014.

[8] J.H. Chon, G. Zarbis-Papastoitsis, Advances in the production and downstream processing of antibodies, N Biotechnol, 28 (2011) 458-463.

[9] K. Champion, Madden. H, Dougherty. J, Shacter E, Defining Your Product Profile and Maintaining Control Over It, Part 2: Challenges of Monitoring Host Cell Protein Impurities, BioProcess International, 3 (2005) 52-27.

[10] ICH, ICH Topic Q 6 B. Specifications:Test procedures and Accepttance Criteria for Biotechnological/Bioogical products, 1999.

[11] E.M. Moussa, J.P. Panchal, B.S. Moorthy, J.S. Blum, M.K. Joubert, L.O. Narhi, E.M. Topp, Immunogenicity of Therapeutic Protein Aggregates, J Pharm Sci, 105 (2016) 417-430.

[12] K. Ahrer, A. Buchacher, G. Iberer, D. Josic, A. Jungbauer, Analysis of aggregates of human immunoglobulin $G$ using size-exclusion chromatography, static and dynamic light scattering, J Chromatogr A, 1009 (2003) 89-96.

[13] J. den Engelsman, P. Garidel, R. Smulders, H. Koll, B. Smith, S. Bassarab, A. Seidl, O. Hainzl, W. Jiskoot, Strategies for the assessment of protein aggregates in pharmaceutical biotech product development, Pharm Res, 28 (2011) 920-933.

[14] Sharma. V K, K.D. S, Experimental Detection and Characterization of Protein Aggregates, in: W.W.C.J. Roberts (Ed.) Aggregation of Therapeutic Proteins, John Wiley \& Sons, New Jersey, 2010, pp. 205-256.

[15] Krishnamurthy. R, Sukumar. M, Das. T K, L.N. A, Emerging Analytical Technologies for Biotherapeutics Development., Bioprocess International, DOI ( 2008.) 32-42. 
[16] H. Zhao, M. Diez, A. Koulov, M. Bozova, M. Bluemel, F. Kurt, Characterization of Aggregates and Particles Using Emerging Techniques, in: H.-C. Mahler, W. Jiskoot (Eds.) Analysis of Aggregates and Particles in Protein Pharmaceuticals, John Wiley \& Sons, Inc., New Jersey, 2012.

[17] D.K. Sharma, D. King, P. Oma, C. Merchant, Micro-flow imaging: flow microscopy applied to sub-visible particulate analysis in protein formulations, AAPS J, 12 (2010) 455-464.

[18] A. Pekar, M. Sukumar, Quantitation of aggregates in therapeutic proteins using sedimentation velocity analytical ultracentrifugation: practical considerations that affect precision and accuracy, Anal Biochem, 367 (2007) 225-237.

[19] S.A. Berkowitz, Role of analytical ultracentrifugation in assessing the aggregation of protein biopharmaceuticals, AAPS J, 8 (2006) E590-605.

[20] W.F.t. Weiss, J.P. Gabrielson, W. Al-Azzam, G. Chen, D.L. Davis, T.K. Das, D.B. Hayes, D. Houde, S.K. Singh, Technical Decision Making With Higher Order Structure Data: Perspectives on Higher Order Structure Characterization From the Biopharmaceutical Industry, J Pharm Sci, 105 (2016) 3465-3470.

[21] S.M. Kelly, T.J. Jess, N.C. Price, How to study proteins by circular dichroism, Biochimica et Biophysica Acta (BBA) - Proteins and Proteomics, 1751 (2005) 119-139.

[22] S.M. Kelly, N.C. Price, The application of circular dichroism to studies of protein folding and unfolding, Biochim Biophys Acta, 1338 (1997) 161185.

[23] A. Sinz, Chemical cross-linking and FTICR mass spectrometry for protein structure characterization, Anal Bioanal Chem, 381 (2005) 44-47.

[24] D.J. Houde, S.A. Berkowitz, Biophysical Characterization of Proteins in Developing Biopharmaceuticals, Elsevier 2015.

[25] R.E. Iacob, G.M. Bou-Assaf, L. Makowski, J.R. Engen, S.A. Berkowitz, D. Houde, Investigating monoclonal antibody aggregation using a combination of H/DX-MS and other biophysical measurements, J Pharm Sci, 102 (2013) 4315-4329.

[26] J.P. Renaud, C.W. Chung, U.H. Danielson, U. Egner, M. Hennig, R.E. Hubbard, H. Nar, Biophysics in drug discovery: impact, challenges and opportunities, Nat Rev Drug Discov, 15 (2016) 679-698.

[27] R. Paul, A. Graff-Meyer, H. Stahlberg, M.E. Lauer, A.C. Rufer, H. Beck, A. Briguet, V. Schnaible, T. Buckel, S. Boeckle, Structure and Function of Purified Monoclonal Antibody Dimers Induced by Different Stress Conditions, Pharmaceutical Research, 29 (2012) 2047-2059.

[28] Remtulla. N, Feasibility of microfluidic routes to monitor protein stability as a tool for bioprocessing, Biochemical Engineering, UCL UCL, 2015.

[29] M. Sutter, S. Oliveira, N.N. Sanders, B. Lucas, A. van Hoek, M.A. Hink, A.J. Visser, S.C. De Smedt, W.E. Hennink, W. Jiskoot, Sensitive spectroscopic detection of large and denatured protein aggregates in 
solution by use of the fluorescent dye Nile red, J Fluoresc, 17 (2007) 181 192.

[30] P. Bhoskar, B. Belongia, R. Smith, S. Yoon, T. Carter, J. Xu, Free light chain content in culture media reflects recombinant monoclonal antibody productivity and quality, Biotechnol Prog, 29 (2013) 1131-1139.

[31] M. Schroder, R. Schafer, P. Friedl, Induction of protein aggregation in an early secretory compartment by elevation of expression level, Biotechnol Bioeng, 78 (2002) 131-140.

[32] P. Hossler, S.F. Khattak, Z.J. Li, Optimal and consistent protein glycosylation in mammalian cell culture, Glycobiology, 19 (2009) 936-949. [33] S. Pande, A. Rahardjo, B. Livingston, M. Mujacic, Monensin, a small molecule ionophore, can be used to increase high mannose levels on monoclonal antibodies generated by Chinese hamster ovary production cell-lines, Biotechnol Bioeng, 112 (2015) 1383-1394.

[34] D. Reusch, M.L. Tejada, Fc glycans of therapeutic antibodies as critical quality attributes, Glycobiology, 25 (2015) 1325-1334.

[35] European Medicines Agency, Guideline on development, production, characterisation and specification for monoclonal antibodies and related products, London, 2008.

[36] Z. Zhang, H. Pan, X. Chen, Mass spectrometry for structural characterization of therapeutic antibodies, Mass Spectrom Rev, 28 (2009) 147-176.

[37] I. Burnina, E. Hoyt, H. Lynaugh, H. Li, B. Gong, A cost-effective platebased sample preparation for antibody $\mathrm{N}$-glycan analysis, J Chromatogr $\mathrm{A}$, 1307 (2013) 201-206.

[38] M.A. Bynum, H. Yin, K. Felts, Y.M. Lee, C.R. Monell, K. Killeen, Characterization of IgG $\mathrm{N}$-glycans employing a microfluidic chip that integrates glycan cleavage, sample purification, LC separation, and MS detection, Anal Chem, 81 (2009) 8818-8825.

[39] H. Stockmann, B. Adamczyk, J. Hayes, P.M. Rudd, Automated, highthroughput IgG-antibody glycoprofiling platform, Anal Chem, 85 (2013) 8841-8849.

[40] M. Wuhrer, M.I. Catalina, A.M. Deelder, C.H. Hokke, Glycoproteomics based on tandem mass spectrometry of glycopeptides, J Chromatogr B Analyt Technol Biomed Life Sci, 849 (2007) 115-128.

[41] E.D. Dodds, R.R. Seipert, B.H. Clowers, J.B. German, C.B. Lebrilla, Analytical performance of immobilized pronase for glycopeptide footprinting and implications for surpassing reductionist glycoproteomics, J Proteome Res, 8 (2009) 502-512.

[42] E.C. Stigter, G.J. de Jong, W.P. van Bennekom, Development of an open-tubular trypsin reactor for on-line digestion of proteins, Anal Bioanal Chem, 389 (2007) 1967-1977.

[43] C.A. Srebalus Barnes, A. Lim, Applications of mass spectrometry for the structural characterization of recombinant protein pharmaceuticals, Mass Spectrom Rev, 26 (2007) 370-388. 
[44] M. Jin, N. Szapiel, J. Zhang, J. Hickey, S. Ghose, Profiling of host cell proteins by two-dimensional difference gel electrophoresis (2D-DIGE): Implications for downstream process development, Biotechnol Bioeng, 105 (2010) 306-316.

[45] B. Nogal, K. Chhiba, J.C. Emery, Select host cell proteins coelute with monoclonal antibodies in protein A chromatography, Biotechnol Prog, 28 (2012) 454-458.

[46] A.A. Shukla, C. Jiang, J. Ma, M. Rubacha, L. Flansburg, S.S. Lee, Demonstration of robust host cell protein clearance in biopharmaceutical downstream processes, Biotechnol Prog, 24 (2008) 615-622.

[47] S.X. Gao, Y. Zhang, K. Stansberry-Perkins, A. Buko, S. Bai, V. Nguyen, M.L. Brader, Fragmentation of a highly purified monoclonal antibody attributed to residual $\mathrm{CHO}$ cell protease activity, Biotechnol Bioeng, 108 (2011) 977-982.

[48] S.K. Fischer, M. Cheu, K. Peng, J. Lowe, J. Araujo, E. Murray, D. McClintock, J. Matthews, P. Siguenza, A. Song, Specific Immune Response to Phospholipase B-Like 2 Protein, a Host Cell Impurity in Lebrikizumab Clinical Material, AAPS J, 19 (2017) 254-263.

[49] C.E. Hogwood, D.G. Bracewell, C.M. Smales, Measurement and control of host cell proteins (HCPs) in CHO cell bioprocesses, Curr Opin Biotechnol, 30 (2014) 153-160.

[50] P. Picotti, R. Aebersold, Selected reaction monitoring-based proteomics: workflows, potential, pitfalls and future directions, Nat Methods, 9 (2012) 555-566.

[51] S. Gallien, E. Duriez, C. Crone, M. Kellmann, T. Moehring, B. Domon, Targeted proteomic quantification on quadrupole-orbitrap mass spectrometer, Mol Cell Proteomics, 11 (2012) 1709-1723.

[52] L.C. Gillet, P. Navarro, S. Tate, H. Rost, N. Selevsek, L. Reiter, R. Bonner, R. Aebersold, Targeted data extraction of the MS/MS spectra generated by data-independent acquisition: a new concept for consistent and accurate proteome analysis, Mol Cell Proteomics, 11 (2012) 0111 016717.

[53] D.E. Walker, F. Yang, J. Carver, K. Joe, D.A. Michels, X.C. Yu, A modular and adaptive mass spectrometry-based platform for support of bioprocess development toward optimal host cell protein clearance, MAbs, 9 (2017) 654-663.

[54] A. Farrell, S. Mittermayr, B. Morrissey, N. Mc Loughlin, N. Navas Iglesias, I.W. Marison, J. Bones, Quantitative host cell protein analysis using two dimensional data independent LC-MS(E), Anal Chem, 87 (2015) 9186-9193.

[55] A.S. Tait, C.E. Hogwood, C.M. Smales, D.G. Bracewell, Host cell protein dynamics in the supernatant of a mAb producing $\mathrm{CHO}$ cell line, Biotechnol Bioeng, 109 (2012) 971-982.

[56] Q. Zhang, A.M. Goetze, H. Cui, J. Wylie, B. Tillotson, A. Hewig, M.P. Hall, G.C. Flynn, Characterization of the co-elution of host cell proteins with 
monoclonal antibodies during protein A purification, Biotechnol Prog, 32 (2016) 708-717.

[57] J.S. Bee, L. Tie, D. Johnson, M.N. Dimitrova, K.C. Jusino, C.D. Afdahl, Trace levels of the $\mathrm{CHO}$ host cell protease cathepsin $\mathrm{D}$ caused particle formation in a monoclonal antibody product, Biotechnology Progress, 31 (2015) 1360-1369.

[58] Corrêa. D H A, R.C.H. I, The use of circular dichroism spectroscopy to study protein folding, form and function, African Journal of Biochemistry Research, 3.5 (2009) 164-173.

[59] J.L. Cole, J.W. Lary, P.M. T, T.M. Laue, Analytical ultracentrifugation: sedimentation velocity and sedimentation equilibrium, Methods Cell Biol, 84 (2008) 143-179. 


\section{Figures and tables}

\section{Figure 1}

Steps to controlling quality attributes. Schematic of the necessary steps/questions that need to be asked in order to ensure control quality attributes in a process.

\section{Figure 2}

Bioprocess flow diagram, showing the stages during upstream (green) and downstream (blue) processing along with components that are entered and removed from the process.

\section{Figure 3}

(A) Size-based detection ranges of various analytical techniques for aggregation. Data obtained from Sharma and Kalonia 2010; Engelsman et al. 2011. Size Exclusion Chromatography (SEC), Dynamic Light Scattering (DLS), Analytical Ultra centrifugation (AUC), Nanoparticle Tracking Analysis (NTA).

(B) Summary of the different techniques for aggregate characterisation. Data compiled from Sharma and Kalonia [14], Engelsman et al. [13], Kelly et al. [21], Corrêa and Ramos [58], Cole et al. [59]. 


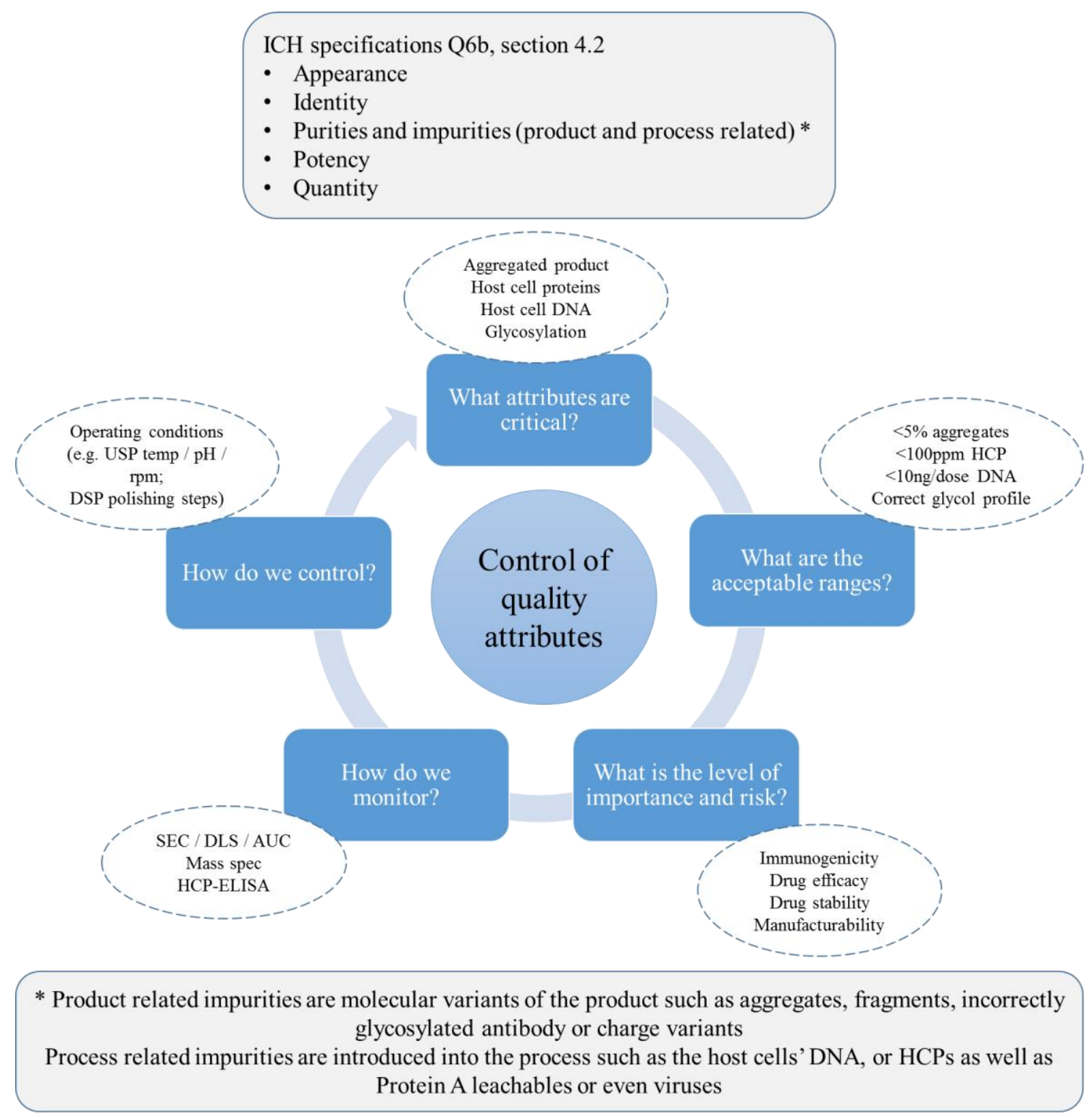

\section{Figure 1}


Components
entering the process

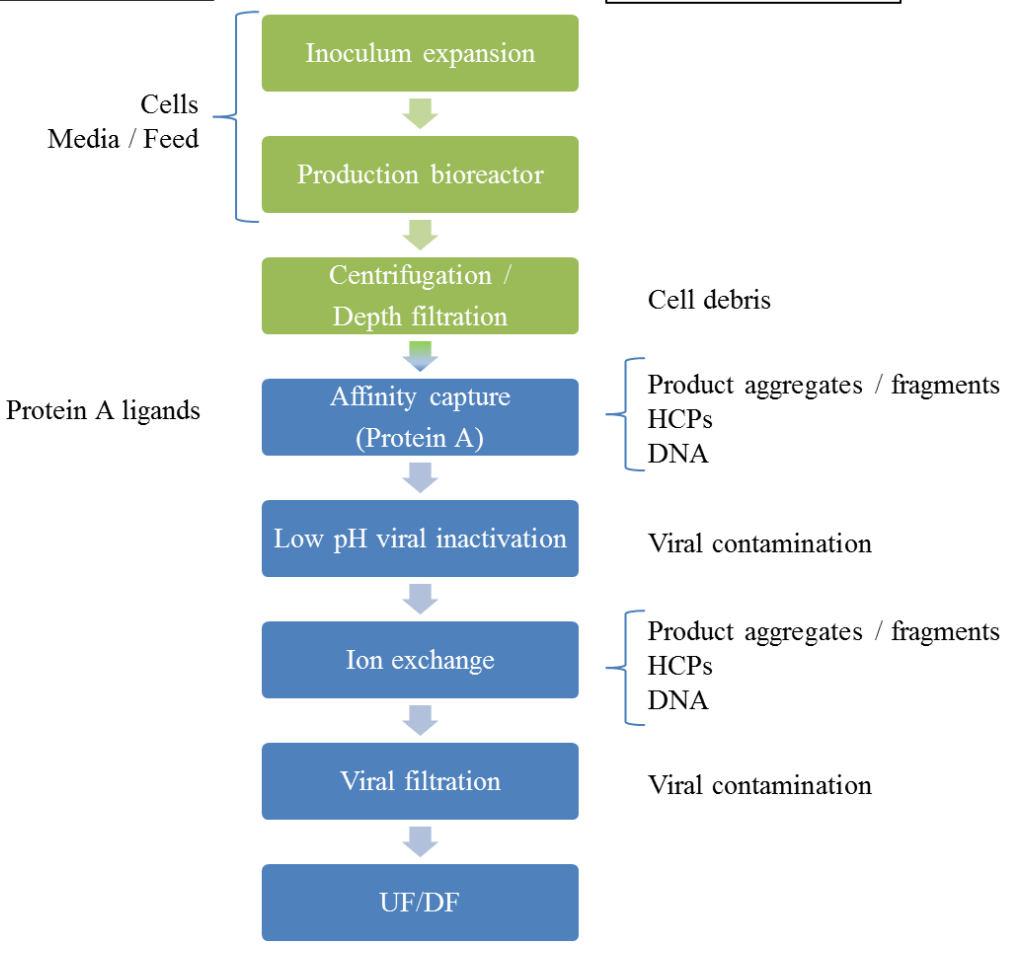

Components removed

from the process

Product aggregates / fragments HCPs

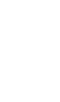


(A)

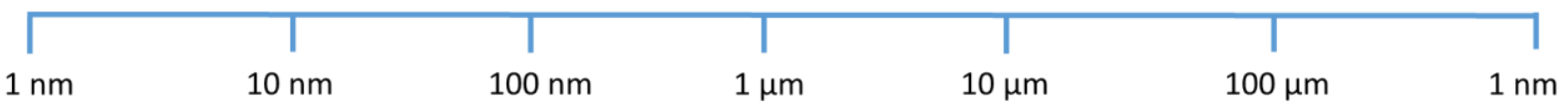

r Monomer _-

(B)

\begin{tabular}{|c|c|c|c|c|c|}
\hline & SEC & DLS & AUC & CD & Fluorescence \\
\hline Sensitivity & $\begin{array}{l}\text { High/ } \\
\text { medium }\end{array}$ & High & Low & Low & High \\
\hline $\begin{array}{l}\text { Sample } \\
\text { Throughput }\end{array}$ & Medium & High & Low & Medium & High \\
\hline Focus & $\begin{array}{l}\text { Small } \\
\text { aggregates }\end{array}$ & $\begin{array}{l}\text { Large } \\
\text { aggregates }\end{array}$ & $\begin{array}{l}\text { Small- } \\
\text { intermediate } \\
\text { aggregates }\end{array}$ & $\begin{array}{l}\text { Soluble } \\
\text { aggregates with } \\
\text { alpha helices }\end{array}$ & $\begin{array}{l}\text { Soluble, sub- } \\
\text { visible and } \\
\text { visible } \\
\text { particles }\end{array}$ \\
\hline Sample & $\begin{array}{l}30 \mu \mathrm{l}, \\
1 \mathrm{mg} / \mathrm{ml}, \\
95 \% \text { purity } \\
\text { on Protein A }\end{array}$ & $\begin{array}{l}100 \mu \mathrm{L}, \\
1-10 \mathrm{mg} / \mathrm{ml}, \\
\text { High purity } \\
\text { preferred }\end{array}$ & $\begin{array}{l}400 \mu \mathrm{L}, \\
1 \mathrm{mg} / \mathrm{ml}, \\
>95 \% \\
\text { purity [59] }\end{array}$ & $\begin{array}{l}95 \% \text { purity on } \\
\text { SDS PAGE [21], } \\
1-10 \mathrm{mg} / \mathrm{ml}[58]\end{array}$ & $\begin{array}{l}\text { Purified } \\
\text { sample } \\
\text { preferred }\end{array}$ \\
\hline
\end{tabular}

Figure 3 


\begin{tabular}{lll}
\hline Technique & Advantages & Disadvantages \\
\hline ELISA & Highly sensitive \& fast & Only semi-quantitative and \\
& measurements of HCP amounts & unable to identify HCP species \\
\hline 2D-PAGE or & Visualises HCPs and provides & Abundant proteins mask less \\
2D-DIGE & information about molecular & abundant ones and make \\
& weight and isoelectronic point & detection of the latter impossible \\
\hline LS-MS/MS & Identifies HCP species of & Takes a lot of time and work \\
(coupled with 2D & interest & \\
electrophoresis) & & Only works on immunogenic \\
\hline Western blotting & Confirms presence of (specific) & HCPs for which antibodies are \\
& HCP species & available; can be expensive \\
\hline
\end{tabular}

\section{Table 1}

Advantages and disadvantages of various analytical tools for the measurement of host cell protein and aggregate impurities. 